anthropology \& materialism

\section{Anthropology \& Materialism}

A Journal of Social Research

$2 \mid 2014$

The Persistence of Myth

\title{
Zehn Thesen zur wirklichen Bewegung des freien Vereins
}

Dix thèses sur le mouvement réel de la libre association

\section{Jan Rolletschek}

\section{(2) OpenEdition}

Journals

\section{Electronic version}

URL: http://journals.openedition.org/am/340

DOI: $10.4000 / a m .340$

ISSN: 2364-0480

\section{Publisher:}

CETCOPRA, CRASSH - Center for Research in the Arts Social Sciences and Humanities, Fakultät Gestaltung - Universität der Künste Berlin

\section{Electronic reference}

Jan Rolletschek, "Zehn Thesen zur wirklichen Bewegung des freien Vereins », Anthropology \&

Materialism [Online], 2 | 2014, Online erschienen am: 15 April 2014, abgerufen am 02 Mai 2019. URL :

http://journals.openedition.org/am/340; DOI : 10.4000/am.340

This text was automatically generated on 2 mai 2019.

Tous droits réservés 


\section{Zehn Thesen zur wirklichen Bewegung des freien Vereins}

Dix thèses sur le mouvement réel de la libre association

Jan Rolletschek

\section{Wenn es überhaupt einen Sinn hat, von einem} Verein freier Menschen zu sprechen, so kann darunter nur eine Verbindung der Leute in Freiheit verstanden werden: die freie Assoziation. Sie gerade ist heute am fraglichsten

1 In der linken politischen Philosophie herrscht nahezu Einigkeit in der Zurückweisung des freien Vereins. Neo-Leninismen und Neo-Heideggerianismen reichen sich die Hände und bilden ein artikuliertes Ganzes. Ihr Konvergenzpunkt ist die Affirmation des Staates, negativ ausgedrückt: die Denunziation der freien Assoziation, einer Organisation durch Räte, als demokratische Illusion. Stattdessen findet man sich, wie überall sonst, in die Komplementarität von Regierung und Opposition. Man zieht seine Lehren aus der Dekonstruktion, indem man die verdinglichte Herrschaft ontologisch verewigt. Oder aber man weist ihre Lehren zurück und träumt den neo-maoistischen Traum eines Bonapartismus von Links. So oder so: Die freie Assoziation soll es nicht geben.

2 Ohne viel Aufsehen nimmt das linke Denken so seinen Platz ein in der Geschichte der politischen Philosophie, die zuvorderst die Geschichte der unterschiedlichen Anstrengungen ist, einer einfachen Schlussfolgerung auszuweichen: der Freiheit der Assoziation. 


\section{Die freie Assoziation ist, in ihrer ersten formalen Bestimmung, die Zustimmung aller zu einer gegebenen Aufteilung, bezogen auf den Gesamtzusammenhang wirklich bestehender Verbindungen}

3 Die freie Assoziation ist die Identität von freier, notwendig alegaler Gleichheit und gleicher Freiheit. Denn die Freiheit ist die Freiheit der Zustimmung unter dem Gesichtspunkt der Universalität, das heißt der Gleichheit. Die freie Assoziation ist demnach die freie Zustimmung aller zu allem im Bereich des wirklichen Zusammenhangs. Als solche ist sie ideal, umfassend und - wenn auch unter Umständen möglich (vgl. These 9) - nicht verifizierbar. Denn nirgends präsentieren sich dieses „alle“ und diese Gesamtheit bestehender Verbindungen.

4 Dennoch ist es nötig, gegen die ausgeprägte Neigung der Zeit, die freie Assoziation zunächst in dieser, ihrer Idealität - einer differenziellen, nicht-äquivalenten Gleichheit

- zu bestätigen.

\section{In Bezug auf die Vernunftforderung der freien Assoziation ist ein skeptisches Bewusstsein unmöglich, weil sie aus der Begründungslosigkeit jeder konkreten Form der Vergesellschaftung selbst gefolgert werden muss}

Hinsichtlich der Notwendigkeit der Entscheidung begegnet man oft einer Ebenenkonfusion. Gerade weil keine Entscheidung möglich ist, heißt es dann, sei eine solche notwendig. So wird der Autoritarismus begründet. Aber diese Begründung ist nichts anderes, als die an das Denken ergehende Aufforderung abzudanken. Was mit logischer - nicht historischer - Notwendigkeit aus der negativen Unmöglichkeit der Entscheidung folgt, ist indes die positive Konsequenz der freien Assoziation. Beide sind wie Vorder- und Rückseite einer Münze. So identifiziert sich, im besten Sinne, das Ende der großen Erzählungen mit der großen Erzählung vom Ende der Vorgeschichte. Erst oberhalb dieser Konsequenz kann vernünftigerweise von Entscheidungen gesprochen werden.

6 Man weiß, dass aus Zweifel Gewissheit erwachsen kann, und Orientierung. Und so gibt es auch hinsichtlich der freien Assoziation keinen Zweifel. Es gibt nur Vernunft oder ihre Verdunklung, Anarchie oder Autorität.

\section{Solange nicht jede/r Einzelne frei ist, gibt es Freiheit, die nicht Unterdrückung ist, nur als Befreiung}

7 Da die Freiheit in der freien Assoziation universal und deshalb mit Gleichheit identisch ist, ist sie nicht auf Unfreiheit gegründet. Sie heißt zum Unterschied von jener Freiheit, die Unfreiheit zur Bedingung hat, Autonomie. Freiheit von Unterdrückung ist notwendige 
Bedingung der freien Zustimmung. Sie gibt der ersten völlig leeren Bestimmung der freien Assoziation, als Zustimmung aller zu allem, ihren Inhalt. In der Ausdehnung des wirklichen Zusammenhangs gibt es Autonomie im vollen Sinne für jede/n Einzelne/n erst, wenn es sie für alle gibt. Das heißt, dass es Freiheit als Autonomie vor dieser Zeit nur als eine ausgleichende Bewegung der Befreiung geben kann. Ebenso kann, was nicht Befreiung ist, nur als Unterdrückung (aktiv oder passiv) gelten. Gegen diesen Gedanken bemüht man heute gern die angeblich kurze Reichweite menschlicher Empathie. Aber wohin die Affektkraft der Vernunft nicht als Empathie reicht, reicht sie allemal als Ekel vor solchem Räsonnement.

8 Freiwillig oder nicht bleiben die Menschen einander verbunden. So lange nicht alle frei sind, kann Autonomie folglich nur Befreiung sein.

\section{Der Weg der Befreiung jeder/s Beliebigen ist der Weg der Befreiung aller}

Wenn niemand in vollem Sinne autonom ist, so lange nicht alle es sind und daher recht verstandene Autonomie einstweilen nur Befreiung sein kann, bedeutet dies auch, dass die Selbstbefreiung aus Unterdrückung zugleich auch Weg der Befreiung aller und das Subjekt dieser Befreiung beliebig ist. Die so verstandene Selbstbefreiung der Expropriateure etwa wäre ihre Selbstexpropriation. Die Befreiung der „Arbeiterklasse“ wäre nicht weniger Sache der „Kapitalisten“ als der Arbeiterklasse selbst, weil sie einfach die Sache aller ist. Das Subjekt der wirklichen Befreiung formiert sich nicht a priori, sondern in einer Praxis, die es zugleich als Klasse entsubjektiviert.

10 Für die Zusammensetzung des Subjekts bedeutet dies, dass es aus lauter Beliebigen besteht. Die Herkunft zählt für nichts. Die Praxis ist alles. Es ist überzählig in Bezug auf die Situation ganz allein aus dem Grund, dass es diese missbilligt und verändern will, d.h. verändert.

\section{Die freie Assoziation als wirkliche Bewegung bedarf einer positiven Entwicklung ihrer praktischen Konsequenzen, so wie sie ihre positive Orientierung im Begriff der Autonomie besitzt}

11 Eine bestimmte Negation gibt nicht, gleichsam als Hohlform oder auf ihrer Rückseite, ein bestimmtes Positives frei. Eine Unendlichkeit bleibt unendlich, auch wenn aus ihr gestrichen wird, und ein Positives ist auf diese Weise nicht zu erreichen. Jede nur auflösende Bewegung bleibt ohne Basis und Ausblick, stößt schnell an ihre Grenzen und zerfällt. Hingegen negiert eine bestimmte Affirmation - indem sie ihm die Kraft abzieht - all das, was sie ausschließt. Sie verschwendet ihre Kraft nicht in der Zerstörung und zerstört doch umfassend. Weniger also ist die Negation auch ein umschaffendes Aufbauen, als dass Letzteres auch eine umfassende Negation ist. Was hier erreicht wird, zählt doppelt. Die selbe Bewegung der Hand, die es hier zusammenfügt, zieht es dort ab, und die gewonnenen Freunde fehlen auch noch in den Reihen der Feinde. Das Material und die Kraft sind nicht unendlich; jeder Exodus ist immanent. Er löst nicht aus den Verbindungen, sondern verändert sie. 
Wäre auch die empörte Ablehnung ein Erstes, gebührte doch dem umschaffenden Aufbau, der Affirmation, das praktische Primat. Diese wirkliche Umwälzung der Verhältnisse ist zugleich stiller, schwieriger und unheroischer als eine politische Revolution, die ihre Bedeutung und Dauer doch nur aus dieser vorhergehenden Umwälzung erhalten könnte.

\section{Die freie Assoziation als wirkliche Bewegung ist zu allen Zeiten möglich. Ihre Bedingungen sind die gerade in jedem Moment gegebenen Voraussetzungen}

13 Die freie Assoziation ist niemals noch-nicht oder nicht-mehr möglich, denn sie ist ja gar keine technische oder natürliche Kategorie, sondern eine gesellschaftliche und als solche unabhängig von den äußeren Bedingungen, vom Entwicklungsstand der Produktivkräfte ebenso wie vom Zerstörungsgrad der Natur. Die wirkliche Bewegung nimmt eben die Technik in Gebrauch, die in jedem Moment verfügbar gemacht werden kann. Sie ist überhaupt nur wirklich, insofern sie sich schon-jetzt, unter den jeweils vorgefundenen Voraussetzungen, praktisch realisiert.

14 Sie ist auch kein innerweltliches Jenseits, kein Himmelreich der Freiheit, das sich nach der Zahl der Mußestunden bemisst, während die Stätte der Produktion die ewige Hölle wäre, über deren Pforte geschrieben steht: „Lasst alle Autonomie fahren, die ihr hier eintretet!" Die freie Assoziation ist keine Freizeit. Sie ist ursprünglicher und deshalb umfassender. Worum es ihr als um einen Selbstzweck geht, ist gerade die Freiheit in den Verbindungen arbeitsteiliger Organisation ebenso wie in den Verbindungen, die auch den Bereich der Freiheit von derartiger Notwendigkeit noch durchziehen und auf seine notwendigen Voraussetzungen verweisen.

15 Die freie Assoziation als wirkliche Bewegung existiert nur, insofern sie sich in den wirklich bestehenden Verbindungen der gesellschaftlichen Produktions- und Reproduktionsprozesse schon jetzt realisiert.

\section{Die wirkliche Bewegung der freien Assoziation existiert nur, insofern sie der Bewegung des Kapitals entgegengesetzt ist}

16 Während des Neunzehnten und Zwanzigsten Jahrhunderts hat die kommunistische Bewegung ihr Schicksal an die Bewegung des Kapitals geknüpft. Doch nichts an dieser Bewegung treibt über sie selbst hinaus. Die Dynamik des Kapitals bietet nur eine ganz negative Orientierung. Die verzweifelte Auffassung, die Arbeiterschaft schwimme mit dem Strom und es fehle je nur ein letztes, um irgend ihr objektiv schon zuträgliche Tendenzen der kapitalistischen Entwicklung zu verwirklichen, ist vielleicht das größte Hindernis, das der Marxismus ihrer Befreiung in den Weg legen konnte. Die Erzählung einer historischen Notwendigkeit des Kommunismus war nie etwas anderes als Erbauungsprosa, eine Art Opium des Proletariats. Gläubige Adaptation bürgerlicher Fortschrittsideologie, die auch im offensichtlichen Verfall den Aufstieg wahrsagen, in der Vertiefung der Niederlage noch Anzeichen des Sieges sehen wollte, war sie selbst nur Anzeichen von Verzweiflung. 
17 Subsumierte die Bewegung des Kapitals alles unter sich, wäre die wirkliche Bewegung der freien Assoziation nicht möglich. Wenn sie dennoch keine Donquichotterie ist, dann nur, weil sie gesellschaftliche Reproduktionsprozesse ausweiten kann, die sich dem Kapitalverhältnis entziehen. Unter den Bedingungen seiner Dominanz ist dies allemal ein Kreuzen gegen den Wind.

\section{Wenn die Freiheit der Assoziation heute einen Sinn haben soll, so ist es nötig, sie als eine doppelte Freiheit zu erkennen. Die freie Assoziation - als differenzielle Gleichheit und Zustimmung aller (vgl.} These 2) - wäre demnach befreit auch noch vom Ideal ihres Endes und so zugleich vom Eifer der Schließung. Dies ist ihre zweite formale Bestimmung

18 Die politische Philosophie ist sich nahezu einig, wenn es darum geht, einen stabilen Zustand herbeizuwünschen, wenn es also darum geht, zum Ende zu kommen. Dies ist der Punkt, an dem sie je schon mit dem Staat paktiert. Die freie Assoziation hingegen ist befreit vom Phantasma des Zustands und so auch vom Eifer der Schließung. Sie will zu keinem Ende kommen, sondern weiß sich als wirkliche Bewegung ohne Ende.

19 Die Lehren der Dekonstruktion auf diese Weise zu ziehen, bedeutet indes nicht, dass die freie Assoziation nicht erreicht und, wie ein Ideal, nur immer angenähert werden könnte. Es bedeutet vielmehr, dass sie nur erreicht werden kann, wenn sie doppelt frei, befreit ist auch noch vom Eifer der Stillstellung ihrer eigenen wirklichen Bewegung und Organisation. Als solche ist sie tatsächlich ganz und gar möglich: das umfassende Ende des Staates und jeder Herrschaft. Ich schlage deshalb vor, den Inhalt dessen, was man traditionell als einen Verein freier Menschen bezeichnet, zu erweitern und der Freiheit der Assoziation diese zusätzliche Bedeutung der Freiheit vom Fluch des Einen beizulegen.

\section{Die Fassung der freien Assoziation als Bewegung ohne Ende impliziert notwendig eine Reflexivität, die den Dissens ins Zentrum der Assoziation selbst einführt, die sich dennoch nicht trennt}

21 Folgt man rückwärts dem Zeitstrahl der kommunistischen Bewegung, wie sie historisch dominierte, findet man nicht hier oder dort ein besseres Bild des Kommunismus oder den Punkt, an dem die Dinge zum ersten Mal aus dem Ruder gelaufen sind, sondern die Notwendigkeit des Gewordenen. Man kann beliebig weit zurückgehen. Es gibt keine Reinheit des Ursprungs. Vielmehr wird man sehen, dass dieser selbst geteilt ist und die kommunistische Bewegung sich an jeder ihrer Stationen vielleicht wesentlicher noch als gegen einen äußeren, gegen einen inneren Feind konstituiert hat. Die identitäre Konstitution einer Organisation und die Serie ihrer Schismen sind zwei Seiten ein und desselben Prozesses. 
Die freie Assoziation hingegen hätte die Teilung, den Dissens, in sich selbst aufzunehmen. Sie hätte in ihrer Dissensualität zusammen zu sein, anstatt sich der Dynamik der Ausschlüsse zu überlassen, das heißt, sich unter dem Phantasma des Einen in einen prinzipiell endlosen Prozess der „Säuberungen“ zu stürzen. Erst wo die wirkliche Bewegung freier Assoziation sich zwischen Schisma und Konsens in der Mitteilung ihrer Differenzen aufhält, wäre sie entbunden vom Eifer der Schließung und befreit zur Entfaltung ihrer organisatorischen Kraft.

\section{INDEX}

Mots-clés: philosophie politique, démocratie

Keywords: political philosophy, democracy

\section{AUTHOR}

\section{JAN ROLLETSCHEK}

Humboldt-Universität Berlin 